\title{
Cumulative antibiotic use significantly decreases efficacy of checkpoint inhibitors in patients with advanced cancer-reply to the editors
}

\author{
Nadina Tinsley ${ }^{1}$, Donna M. Graham ${ }^{1,2}$, Natalie Cook $^{1,2}$ \\ ${ }^{1}$ The Christie NHS Foundation Trust, Manchester, UK; ${ }^{2}$ Division of Cancer Sciences, University of Manchester, Manchester, UK \\ Correspondence to: Dr. Natalie Cook. Honorary Consultant in Medical Oncology, Experimental Cancer Medicine Team (ECMT), The Christie NHS \\ Foundation Trust, Wilmslow Road, Manchester, M20 4BX, UK. Email: Natalie.Cook@Christie.nhs.uk. \\ Provenance: This is an invited article commissioned by the Section Editor Dr. Xiao Li (Department of Urology, Jiangsu Cancer Hospital, Jiangsu \\ Institute of Cancer Research, Nanjing Medical University Affiliated Cancer Hospital, Nanjing, China). \\ Response to: Casadei C, Lolli C, Farolfi A. Immune-checkpoint inhibitors and the importance of concomitant medications: focus on antibiotics. Ann \\ Transl Med 2019;7:S339. \\ Reed JP, Devkota S, Figlin RA. Gut microbiome, antibiotic use, and immunotherapy responsiveness in cancer. Ann Transl Med 2019;7:S309.
}

Submitted Nov 15, 2019. Accepted for publication Nov 29, 2019.

doi: 10.21037/atm.2019.12.06

View this article at: http://dx.doi.org/10.21037/atm.2019.12.06

We thank the editors for their constructive comments on our recently published article "Cumulative antibiotic use significantly decreases efficacy of checkpoint inhibitors in patients with advanced cancer" (1-3).

We agree with the editorial commentaries discussing substantial progress in identifying the immune system as a significant hallmark in patients with cancer. Immune checkpoint inhibitors (ICI) act by modulating co-inhibitory T-cell signalling and are now commonly used for the treatment of certain cancers, however responses remain varied and heterogeneous. As highlighted, identifying predictive biomarkers has proved challenging in this cohort of diverse patients, with a number of non-validated biomarkers being suggested. With the increasing use of ICI in clinical trials and in other tumour sites, it remains important that we try and understand the interplay between factors that may modify immunotherapy responses $(2,3)$.

In our retrospective study of patients with advanced cancers treated with ICI, we determined that antibiotic use is an independent predictor of shorter progression free survival (PFS) and overall survival (OS). Further explorative analysis was undertaken into single versus cumulative use of antibiotics. This analysis identified an interesting perspective, demonstrating the negative effect of antibiotics on PFS and OS is significantly enhanced with cumulative use.

Therefore, as with other studies, we determine that antibiotic use appears to have a deleterious effect which is enhanced with cumulative use (4-7).

The editorial authors point out the timeframe for analysis between ICI initiation and antibiotic use in our study concentrates on the later effects of antibiotic use (6 weeks post-ICI initiation). This is reflected in the patient sample with a higher proportion of these patients having antibiotic treatment. Currently available evidence provides no certainty as to when antibiotics exert their highest deleterious effect on the microbiome and how long this takes to recover its natural flora. Effects can normally be seen within days of antibiotic exposure but may persist for several months to years after, such as in peptic ulcer eradication therapy where the desired effect is to eradicate Helicobacter Pylori (8). We agree with the editors that this requires more clarity as there are inconsistencies in the time frames of antibiotic therapy assessed across studies. A recently published study questioned the effect of antibiotic treatment concurrently with, or prior to, commencement of ICI and demonstrated a significant detrimental impact of pre-ICI antibiotic use (9). As suggested by Reed and colleagues (2), acquiring better understanding of the most impactful timeframe for antibiotic-induced dysbiosis would provide guidance to better equip clinicians in optimising patient benefit from ICI. However, further prospective information is required to understand the interactions 
between other concomitant medications and comorbidities in this setting, where polypharmacy is often an issue. Limited sample data, which included a lack of isolated organism in all cases, prevented exploration of the effect of pre-ICI and post ICI antibiotic therapy and correlations between specific antibiotic classes in our study.

The lack of microbiome analysis in our retrospective study prevented any mechanistic understanding of the detrimental impact of antibiotic use on ICI outcomes. Based upon preclinical and emerging clinical evidence, we postulated that this may be related to antibioticrelated microbiota (4). Casadei and colleagues explore the possibility that an exhausted immune system may result in more infections requiring antibiotic use (3). Investigation of these questions necessitates a prospective approach, beyond the scope of this real-world data analysis. Retrospectively grading severity of infections from commentary in patient's medical notes may lead to interpretation bias and inaccuracies. Nonetheless, we feel that this work adds to the growing body of literature that is published in this area, helping to further understand the role of single and cumulative antibiotics use in this cohort of patients.

We recognise that this retrospective analysis of a single site will invariably have limitations. However, inclusion in this study was not restricted to a particular subtype of malignancy and reflects the variation of patients presently receiving immunotherapy in routine clinical practice or clinical trials. Independent database verification was undertaken to minimise inconsistencies with a rigorous approach adopted for calculating PFS and OS to ensure that no bias was introduced.

The editorial commentaries present various other perspectives and raise questions about how this growing body of knowledge can be interpreted and applied clinically. Indeed, research in mice has shown how antibiotics can directly remodel the biochemical environment of cells during infection and lower immune function by affecting the host microenvironment (10). This potentially presents patients who require antibiotic treatment as a more vulnerable group of patients and highlights the multifariousness of factors that may influence complex ICI responses. The evidence that antibiotics lower ICI responses needs to be carefully evaluated and applied on an individual basis, whilst recognising that sepsis remains a leading cause of death in patients with cancer. Antibiotic use remains recommended in patients were an infection is suspected. The duration of antibiotics should be carefully considered, possibly with expertise sought from
Microbiology specialists. In some cases, in may be prudent to delay the start of ICI initiation, which theoretically can allow for the microbiome to recover, maximising the chance of response. However, this approach is not currently supported by evidence and may risk deterioration with disease progression in a proportion of patients. Preclinical and clinical investigation of a safe antibiotic window and potential mitigation strategies prior to commencing ICI should be investigated. Complex decision making may be needed in patients who require antibiotics for recurrent infections and optimal management if ICI is offered. Without the benefit of prospective data, it is currently not clear and potentially unsafe to implement specific guidelines on antibiotic use in patients treated with ICI, as case-bycase expertise is required to avoid detrimental outcomes of infection. Nonetheless, restrictions on corticosteroid use in ICI patients are in place after studies found a similar detrimental outcome, meaning that with increasing use of real-world, trial data and prospective analyses there may be scope to develop informed guidance in the future.

\section{Acknowledgments}

None.

\section{Footnote}

Conflicts of Interest: The authors have no conflicts of interest to declare.

Ethical Statement: The authors are accountable for all aspects of the work in ensuring that questions related to the accuracy or integrity of any part of the work are appropriately investigated and resolved.

\section{References}

1. Tinsley N, Zhou C, Tan G, et al. Cumulative Antibiotic Use Significantly Decreases Efficacy of Checkpoint Inhibitors in Patients with Advanced Cancer. Oncologist 2020;25:55-63.

2. Reed JP, Devkota S, Figlin RA. Gut microbiome, antibiotic use, and immunotherapy responsiveness in cancer. Ann Transl Med 2019;7:S309.

3. Casadei C, Lolli C, Farolfi A. Immune-checkpoint inhibitors and the importance of concomitant medications: focus on antibiotics. Ann Transl Med 2019;7:S339. 
4. Routy B, Le Chatelier E, Derosa L, et al. Gut microbiome influences efficacy of PD-1-based immunotherapy against epithelial tumors. Science 2018;359:91-7.

5. Derosa L, Routy B, Kroemer G, et al. The intestinal microbiota determines the clinical efficacy of immune checkpoint blockers targeting PD-1/PD-L1. Oncoimmunology 2018;7:e1434468.

6. Pinato DJ, Howlett S, Ottaviani D, et al. Antibiotic treatment prior to immune checkpoint inhibitor therapy as a tumor-agnostic predictive correlate of response in routine clinical practice. J Clin Oncol 2019;37:abstr 147.

7. Hakozaki T, Okuma Y, Omori M, et al. Impact of prior

Cite this article as: Tinsley N, Graham DM, Cook N. Cumulative antibiotic use significantly decreases efficacy of checkpoint inhibitors in patients with advanced cancer-reply to the editors. Ann Transl Med 2020;8(4):139. doi: 10.21037/ atm.2019.12.06 antibiotic use on the efficacy of nivolumab for non-small cell lung cancer. Oncol Lett 2019;17:2946-52.

8. Modi SR, Collins JJ, Relman DA. Antibiotics and the gut microbiota. J Clin Invest 2014;124:4212-8.

9. Pinato DJ, Howlett S, Ottaviani D, et al. Association of Prior Antibiotic Treatment With Survival and Response to Immune Checkpoint Inhibitor Therapy in Patients With Cancer. JAMA Oncol 2019. [Epub ahead of print].

10. Yang JH, Bhargava P, McCloskey D, et al. AntibioticInduced Changes to the Host Metabolic Environment Inhibit Drug Efficacy and Alter Immune Function. Cell Host Microbe 2017;22:757-765.e3. 